\title{
Inferring Health Metrics from Ambient Smart Home Data
}

\author{
Lorcan Walsh, Andrea Kealy, John Loane, Julie Doyle, and Rodd Bond \\ CASALA and the Netwell Centre, \\ Dundalk Institute of Technology, \\ Ireland. \\ Email: $\{$ first name $\} .\{$ last name $\} @$ CASALA.ie
}

\begin{abstract}
As the population ages, smart home technology and applications are expected to support older adults to age in place and reduce the associated economic and societal burden. This paper describes a study where the relationship between ambient sensors, permanently deployed as part of smart aware apartments, and clinically validated health questionnaires is investigated. 27 sets of ambient data were taken from a 28 day block from 13 participants all of whom were over 60 years old. Features derived from ambient sensor data were found to be significantly correlated to measures of anxiety, sleep quality, depression, loneliness, cognition, quality of life and independent living skills (IADL). Subsequently, linear discriminant analysis was shown to predict participants suffering from increased anxiety and loneliness with a high accuracy $(\geq 70 \%)$. While the number of participants is small, this study reports that objective ambient features may be used to infer clinically validated health metrics. Such findings may be used to inform interventions for active and healthy ageing.
\end{abstract}

\section{INTRODUCTION}

The world's population is ageing as a result of changes in life expectancy, fertility and migration, and this demographic shift is set to intensify over the next forty years [1]. This will be most acute in North America, Europe and Japan and is set to place an enormous economic and societal burden on both health and social care systems [1]. Ongoing research proposes a move towards technologies which deliver and support independent living allowing the older adult to age in place for as long as possible and in their own home. Recent advances in health technology have focused on a move towards clinic, community and home-based provision of care. Examples of research in this area include technologies to support clinical assessments (for example, enhanced assessment through the instrumentation of standard physical tests [2]), technologies to support community healthcare providers (for example, a platform to inform a public health nurse of changes in the health status of a patient [3]), and home-based health technology which itself can be multifactorial. Such technologies can be condition specific in nature, meeting certain clinical needs and ranges widely from blood glucose monitors, electronic weighing scales and falls pendant alarms to technologies that alert care providers when unsafe house exits occur (for example in the case where a person with dementia leaves the house late at night/early morning) and applications which alleviate social isolation. However, aggregating data from multiple devices across such an instrumented environment, also known as a smart home, may offer the predictive capacity of inferring both current and future health states.
The concept of smart homes enabling older adults to agein-place is relatively recent. Studies have ranged in design from short stays in highly sensed artificial residences to extended deployments of smart home technologies in the permanent homes of older adults. Often these studies record the daily patterns and behaviours of its residents through a number of sensors, and intelligent algorithms have been created which automatically identify these behaviours using some sort of ground truth measurement. As study durations are extended the sensing topologies generally become less intrusive. For example, the expected adherence rates of older adults in wearing and maintaining (charging, etc) on-body sensors over extended periods is low, especially when there is no perceived benefit. A brief overview of some such smart home health technology deployments is given below.

TigerPlace is a series of 32 private apartments each of which consists of a network of wired and wireless sensors that monitor proximity, motion, listen for audio alerts made by the residents, measure vital signs using multiple sensors (including bed sensors), track residents gait using the Microsoft Kinect platform, and detect cooking patterns using temperature sensors [4]. This research investigates the assessment and prediction of functional decline and the alerting of adverse health events, using these smart home sensors, to care providers. The GatorTech Smart House is a smart home supporting independent living and facilitating the remote monitoring of patients [5]. It combines a large number of sensors including under-floor pressure sensors, RF localisation systems (worn by occupants), a smart mailbox, automatic front door unlocking (for residents), automatic blinds, a smart bed (for extracting bed-restlessness and sleeping patterns), an interactive mirror for communicating with residents (particularly for medication prompting), and smart services for food preparation. The CASAS Smart Home Project is a three-bedroom apartment testbed whose priority is to improve the comfort, safety and/or productivity of the its resident(s) [6]. Data are recorded from motion sensors placed approximately 1 metre apart throughout the entire house, temperature, water and stove usage sensors, phone records, and contact switch sensors, usage sensors on the medicine container and other important ingredients. Models are generated from this data to recognise activities to build behavioural profiles [6], [7], [8]. An important aim of the CASAS Smart Home is to perform functional health assessments longitudinally using ambient sensors, to assess the resident-acceptance of the technologies, and to evaluate the benefit to caregivers. In the ORCATECH Living Lab, a group of community dwelling older adults have unobtrusive 
monitoring technologies installed in their homes since 2006 [10]. Thesse technologies include motion sensors, contact sensors (placed on doors and cabinets), bed movement sensors, motion-based gait speed detector, phone sensors and medication usage detectors. Regular clinical and neuropsychological tests are performed by the 30 participating seniors. In addition, the characteristics of their home environment (type of home, home construction materials, number of rooms, etc.), and of their socialization habits (regular outings and visitors) are documented. Every subject also completes a short weekly online questionnaire. Subsequently, these technologies were rolled out into the homes of 265 older adults in the Intelligent Systems for Assessing Aging Changes (ISAAC) study, and concomitant health and functional questionnaires, physical examinations, and neuropsychiatric testing [10]. Initial results demonstrate the feasibility of the large-scale community deployment of in-home activity assessment technology. The information extracted from the technologies deployed into the homes of these older adults may be used to both inform clinicians of a change in health status, and to connect older adults with a remote health coach and remote family members [9]. Robben and Krose investigated the relationship between ambient sensor data and a functional health assessment [11]. For this study a wireless sensor network was deployed throughout the homes of 9 older adults and compared to results from a functional health assessment completed at three month intervals. While the number of participants was small, significant associations were found.

Great Northern Haven (GNH) is a development of 16 smart aware homes where over 100 ambient sensors and actuators are installed in each apartment. Older adults have permanently occupied fifteen of the apartments since June 2010 with the remaining apartment used for research and demonstration purposes. These apartments are the permanent homes for each resident and as such extremely rich data is being collected. GNH is not a testbed for research but rather the real homes of older adults ageing in place. The collection of such data over extended periods provides a means to understand the behavioural changes of older people as they age within their own homes. In this paper, the relationship between the data collected from the ambient sensors in GNH and its relationship to the health status of residents, as determined by a battery of clinical health questionnaires, is investigated. In particular, the accuracy, sensitivity and specificity of inferring anxiety, poor sleep, and loneliness from a combination of these ambient data is reported upon.

\section{GREAT NORTHERN HAVEN}

The constellation of sensors within Great Northern Haven includes passive infrared (PIR) sensors which trigger when movement occurs, contact sensors which notify when a door opens, light switch sensors water and electricity sensors, brightness sensors and temperature sensors as shown in Firgure 1. The sensors under analysis in this study and their location within each apartment is given in Table I.

A Konex (KNX) building management system is used to connect each of the sensors, identified by a unique id, to a central server within the GNH complex. For each sensor firing, the time, sensor id and value are recorded into a local MSSQL database. Sensor firings occur either when triggered (change in its internal value from 0 to 1 or vice-versa) and/or periodically (for example, to ensure the sensor is working correctly). Subsequent processing attaches the sensor type (eg. kitchen PIR sensor) and a de-identified apartment index to each sensor firing. The relationship between the apartment identifier and the apartment number is only available to limited number of researchers within the research group.

The context aware brokering and inferencing engine (CABIE) is a cloud-based system which analyses data collected from agnostic sensors and facilitates the derivation of higher level information from the raw sensor data. Within this study, CABIE is used to import the sensor data every 15 seconds to enable near real-time processing of streaming data. While low level information may be processed directly from the incoming data (for example, the number of sensor firings per day), CABIE allows higher level processing using a ruby-onrails environment. This allows more complex algorithms which require data from multiple sources to run. For example, the determination of room-level location of the individual derived using an algorithm based on data from a mixture of PIR, door contacts and light switches as well as some deterministic logic [12].

\section{STUDY DESIGN}

Data were extracted from a 28 day period across four distinct data collection points July 2012, November 2012, February 2013 and November 2013. Ethical approval for the collection of ambient smart home data for the GNH development and for the collection of questionnaire data was granted by the ethical review board at Dundalk Institute of Technology and the Health Service Executive, Ireland. Each 28 day period consisted of four weeks consisting of equal numbers of weekdays and weekends, should any change in patterns exists between these periods. At least four months was kept between data collection points from each individual. 33 sets of data for one month were available for analysis, however 7 sets of these data were from dual occupancy apartments and these were not included in any analysis. This resulted in a total of 27 sets of data from 13 participants for this analysis. All data were considered independent for this analysis. Of the 13 participants, 6 were female. Participants were born between 1925 and 1953 corresponding to an age of between approximately 60 and 88 years old.

\section{A. Health Questionnaire Data}

Health questionnaires were administered to the residents of Great Northern Haven, upon consent to take part in the research, guided by an experienced researcher where necessary. Ambient data was selected within two weeks of when these were administered. Cut-off values, taken from normative values for each scale, were used to differentiate between different health statuses as indicated. The following standardised health questionnaires were used:

- Anxiety: HADS ( $\geq 8$ - anxious state)

- $\quad$ Sleep Quality: PSQI ( $\geq 5$ - poor sleeper)

- Depression: CES-D ( $\geq 15$ - depressed)

- Loneliness: De Jong Giervald ( $\geq 3$ - lonely) 


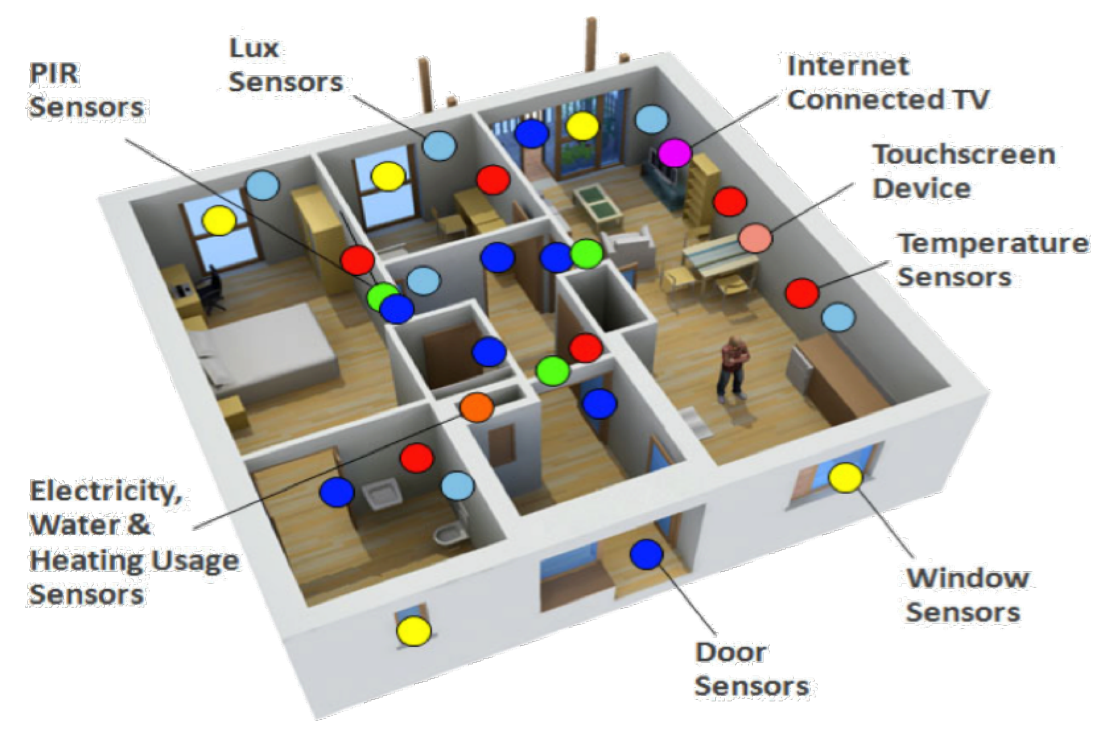

Fig. 1. Great Northern Haven Sensor Constellation.

TABLE I. Great Northern Haven SENSOrS

\begin{tabular}{rrrr}
\hline Sensor type & PIR sensor & Light switch sensor & Door contact sensor \\
\hline & Living Room & Water Closet & Front Door \\
& Hall & Living Room & Rear Door \\
& Bedroom & Kitchen & Living Room \\
& & Hall & Main Bed. Door \\
& & Main Bedroom & En Suite Door \\
Location & & 2nd Bedroom & En Suite \\
& & Sensor value interpretation \\
Sensor reset (0) & No movement & Switch turned off & Door Closed \\
Sensor fired (1) & Movement detected & Switch turned on & Door open \\
\hline
\end{tabular}

- Cognition: MOCA

- Quality of Life: SF-36

- Independent Living Skills: IADL

\section{B. GNH Ambient Features}

Firstly some notation is considered, a binary matrix, $\mathbf{X}$ containing the value outputted by each sensor, is defined as

$$
\mathbf{X}=x(k)_{j}
$$

where $x(k)$ is value of the $j$ th sensor for each sensor firing, $k$. $\mathbf{X}$ may consist of null values at time instants where sensor firings for specific firings did not occur. Each sensor within GNH is triggered upon user activity and as such occurs at inconsistent times. The value of $x(k)$ corresponds to either 1 or 0 as per Table I. The associated time at which each sensor firing occurred was also recorded.

Data for each sensor for each monitoring period was extracted and analysed. A number of features were extracted over this 28 day monitoring period. These features were combined to provide a description of the behavioural patterns of each resident.

The following features were extracted from the raw ambient GNH data:
1) Sensor Firings: The number of firings for each of the door contact sensors, light switch sensors and PIR sensors in each apartment over the recording period were counted. A firing was defined as $x(k)$ changing from a 0 to a 1 .

2) Percentage of time spent in each room/location: A deterministic model was generated using data from the PIR, door and window contact, and light switch sensors to infer the location of the individual within the apartment [12]. The locations (l) of interest are the kitchen/living room, main bedroom, en suite, water closet, second bedroom, hall and outside the house as defined by $\mathrm{l}=1(\mathrm{k})$ where 1 is the location of the individual at sample $\mathrm{k}$.

3) Number of transitions between locations: The number of transitions, T, between locations is defined as the number of samples in the location vector, 1 . The location vector is only updated when a person moves from one location to another.

4) Number of firings for each sensor: The number of firings, F, for each PIR, door contact sensor and light switch sensor, as given in Table 1, was calculated as

$$
F=\Sigma_{j=1}^{M} x(k)_{j}
$$

5) Total duration of activity: The duration a PIR sensor is fired for is given to last a duration $t_{o n}$

$$
t_{o n}(k)=t_{(x(k+1))}-t_{(x(k))}
$$


where the sensor begins firing at time, $t_{(x(k))}$, where $x(k)=1$, until the sensor value returns to zero, $x(k+1)=0$, at time $t_{x(k+1)}$.

The total duration of activity (Total Movement), A, recorded by all PIRs is defined as the sum of time over which the PIR sensor remains at a value of one for all N PIR sensor events.

$$
A=\Sigma_{k=0}^{N} t_{o n}(k)
$$

6) Total duration of nocturnal activity: The total duration of night movement (Nocturnal Movement), NA, recorded by all PIRs is defined as the sum of time over which the PIR sensor remains at a value of one during the night.

$$
N A=\Sigma_{k=0}^{N} t_{\text {on }}(k)
$$

where $t_{o n}(k)$ occurs between 2am and 6am.

\section{ANALYSIS}

Direct pearsons correlation between each of the features derived from the ambient data and the daily health questionnaires were performed (as given in Table II). The ambient features were extracted the 28 day period (for example, the number of PIR firings in the hall or the percentage of time spent in the bedroom) over each of the 27 sets of data and compared to the score for each of the health questionnaires. Significant associations $(p \leq 0.05)$ were found to exist between a number of ambient features and health questionnaires.

Subsequently, it was investigated whether a combination of the ambient features could be used to infer whether a resident of GNH experienced poor sleep, anxiety, depression or loneliness. A linear discriminant classifier was used to perform this classification task where a combination of the principal components are scaled independently to optimally discriminate between participants who either experience each condition or not (e.g. differentiate between good and poor sleepers). For more information on linear discriminant classifiers, the reader is directed to Hastie et al. [13]. Firstly, the ambient health data were normalised (zero mean with unit variance) for each metric. Subsequently, principle component analysis (PCA) was used to reduce the dimensionality of the ambient feature matrix as significant associations were found to exist between the data. The principal components (PCs) that covered $99 \%$ of the variance of the features were included in further analysis. A leave one out cross validation technique was used to provide independent results. In this analysis 27 sets of data were available, 26 of these were used to train the classifier and the remaining data set was used to test the classifier. This was repeated for each set of data and overall performance values were calculated as the mean of each test performance value. The accuracy, sensitivity and specificity results for each classifier for each condition is given in Table III.

\section{RESUlts}

Significant correlations were found to exist between the scores for each of the questionnaires and at most five of the ambient features. These correlations ranged for an absolute $\rho$ value of between 0.343 and 0.788 . While there was no
TABle III. Ambient Health Status Inferencing Classifier RESULTS

\begin{tabular}{rccc}
\hline & Accuracy & Sensitivity & Specificity \\
\hline Poor Sleep & 59 & 70 & 29 \\
Anxiety & 70 & 56 & 78 \\
Depression & 54 & 25 & 67 \\
Loneliness & 73 & 75 & 70 \\
\hline
\end{tabular}

single feature which significantly correlated to all of the health questionnaire scores, the percentage of time spent in the bedroom and living room both correlated to four of the scales. Both correlated to anxiety, IADL and poor sleep, while reduced bedroom time correlated to an increased depression score and increased time spent in the living room was associated with increased loneliness.

The number of PIR firings, the number of transitions between rooms and the total movement (total time active time within the apartment) were not significantly associated with any of the scales. The door contact firings were only associated to one scale for one door where a reduction in front door firings were associated to higher cognition scores. The number of light switch firings, for some locations, were associated significantly with anxiety and inversely correlated to both cognition and IADL (independent living skills).

The nocturnal movement (time spent moving between $2 \mathrm{am}$ and 6am) was inversely correlated with IADL (independent living skills) and positively associated with loneliness.

The percentage of time spent across various locations in the apartments were associated with anxiety, depression, IADL, loneliness and poor sleep. Percentage of time spent in the bedroom was inversely associated with anxiety, depression, IADL and poor sleep. Time spent in the living room was associated with anxiety, loneliness and poor sleep and inversely associated with IADL. Time spent outside was associated with IADL and inversely associated with loneliness.

It was investigated whether a combination of the ambient features could be used to infer anxiety, poor sleep, depression and loneliness, using linear discriminant classification. PCA was used to reduce the dimensionality of the ambient feature data, $\mathbf{X}$, as these data were found to be significantly correlated. Accuracy rates of greater than $70 \%$ were found for the detection of anxiety (sensitivity $56 \%$, specificity $78 \%$ ) or loneliness (sensitivity $75 \%$, specificity $70 \%$ ) using the ambient sensor features. Lower accuracy rates were found for the poor sleep and depression scores (59\% and54\% respectively).

\section{DISCUSSION}

Significant association were found to exist between each of the standardised health questionnaires and the ambient sensor data. The majority of these were related to light switch firings, the percentage of time spent in certain locations throughout the environment, and the levels of nocturnal movement. The light switch firings there positively associated with anxiety, suggesting that more anxious people use light switches more often, and inversely correlated with cognition, suggesting that people with lower levels of cognition tend to switch lights on or off less. 
TABle II. GNH Ambient Data and Health Questionnaire Data Correlation Coefficients (Pearson's R)

\begin{tabular}{|c|c|c|c|c|c|c|c|}
\hline Feature & Location & Anxiety & Cognition & Depression & IADL & Loneliness & Poor Sleep \\
\hline \multirow{5}{*}{ Door Contact Firings } & Bath & 0.05 & -0.229 & 0.2 & 0.284 & -0.307 & 0.068 \\
\hline & Bed1 & 0.018 & -0.297 & 0.282 & 0.229 & -0.18 & -0.123 \\
\hline & Front & 0.128 & -0.518 & 0.093 & 0.206 & -0.11 & -0.181 \\
\hline & Living & -0.25 & -0.087 & -0.367 & 0.226 & -0.333 & -0.33 \\
\hline & Rear & 0.076 & -0.072 & -0.248 & 0.075 & -0.158 & 0.167 \\
\hline \multirow{7}{*}{ Light Switch Firings } & Bath & -0.171 & -0.481 & 0.031 & -0.066 & -0.16 & -0.062 \\
\hline & Bed1 & -0.132 & -0.252 & -0.256 & 0.185 & -0.141 & -0.293 \\
\hline & Bed2 & 0.426 & -0.273 & 0.117 & 0.099 & 0.098 & -0.034 \\
\hline & Hall & -0.218 & -0.626 & -0.065 & -0.003 & -0.092 & -0.338 \\
\hline & Kitchen & -0.07 & -0.12 & -0.235 & 0.222 & -0.17 & -0.258 \\
\hline & Living & 0.118 & -0.576 & 0.111 & -0.343 & 0.363 & 0.17 \\
\hline & WC & 0.429 & -0.312 & 0.316 & -0.144 & 0.305 & -0.018 \\
\hline \multirow{3}{*}{ PIR Firings } & Bed 1 & -0.171 & -0.274 & -0.237 & -0.225 & 0.137 & 0.048 \\
\hline & Hall & 0.017 & -0.373 & 0.012 & 0.063 & -0.123 & -0.149 \\
\hline & Living & 0.007 & -0.101 & -0.098 & 0.163 & -0.07 & 0.192 \\
\hline \multirow[t]{6}{*}{ Number of Transitions } & & 0.109 & -0.193 & -0.078 & 0.07 & -0.043 & 0.009 \\
\hline & Bedroom & -0.564 & -0.24 & -0.418 & -0.402 & -0.015 & -0.398 \\
\hline & En Suite & 0.053 & -0.264 & -0.016 & 0.016 & -0.132 & 0.179 \\
\hline & Hall & 0.118 & 0.261 & 0.119 & 0.339 & -0.19 & 0.316 \\
\hline & Living & 0.456 & 0.076 & 0.297 & -0.548 & 0.788 & 0.397 \\
\hline & Outside & -0.042 & -0.079 & -0.019 & 0.509 & -0.505 & -0.225 \\
\hline Percentage of Time in & WC & 0.227 & -0.138 & 0.382 & 0.081 & 0.069 & 0.097 \\
\hline Nocturnal Movement & & 0.248 & -0.075 & 0.149 & -0.415 & 0.457 & 0.32 \\
\hline Total Movement & & 0.035 & -0.365 & -0.089 & 0.095 & -0.098 & 0.102 \\
\hline
\end{tabular}

This paper proposes a system which can identify individuals with increased anxiety and loneliness using a combination of ambient sensor data and a linear discriminant classifier.. A high accuracy $(\geq 70 \%)$ was reported using a leave-oneout cross validation technique. There was not enough data available to provide fully independent tests of the system. Additionally, the data were treated as independent samples when multiples sets of data from some individuals were used.

This system is based upon those living in the GNH, and as such relates to older adults, the specific configuration of sensors within GNH and the validated feature extraction algorithms applied. However, these results show that ambient sensor data may be used to infer validated clinical health questionnaires, particularly amongst this population.

The data reported upon in this study pertain to a limited number of individuals over multiple data collection points. These are treated independently for this analysis. A more extensive cohort, potentially with repeated sample point, would provide more conclusive evidence of the associations reported in this paper. However, the data analysed is over an extended duration (28 days) and significant periods exist between each data collection point. While it would be ideal to increase the sample population, the GNH is a unique housing development as it is both highly sensed (over 100 sensors and actuators per apartment) and is the permanent homes of the older adult residents who live there. The collection of such data is a difficult process with barriers ranging from engaging and respecting participants and significant technical challenges such as ensuring sensor configurations work continuously and as expected.

This study reports on an initial number of features extracted from ambient sensors deployed throughout apartments within the GNH. There is significant potential to extract further objective features and include them in future studies, for example total sleep time or bathroom visits [12]. While other features may be more difficult to identify from ambient sensors, such as the presence of visitors, however these events may be reflected in some of the existing features (such as the movement levels within the house).

This work excludes the use of subjectively reported data from the participants themselves. For example, participants could self-report on certain scales (e.g. "How rested to you feel?"). Combining subjective and objective data may provide a more holistic representation of an individual's health status. Research investigating the use of a custom health and wellness self-management application is currently underway within CASALA [14]. Aligning both of these systems may aid in the identification of a change in health status and serve as a means to engage the participant in the intervention. The use of subjective data may also provide a means for the participant to provide external context. For example, low activity scores may be recorded however this may be as a result of an external context such as poor weather or as a result of visitors to the environment.

\section{CONCLUSION}

While more extensive research using a larger and completely independent cohort would elucidate the findings further, this paper demonstrates that significant associations exist between objectively measured ambient sensor data and standardised health questionnaires. The data reported upon is collected over extended periods and in the real homes of older adults. It does not pertain to short deployments of sensor kits. Due to the applied and permanent nature of the GNH project, this research recognises the challenges inherent in long-term real-world sensor deployments. Additionally, the nature of the ambient sensor configuration means that participants do not have to consciously engage with the technology making it extremely suitable for extended deployments. 
The system described in this paper may be used to inform interventions for both health and care providers (both formal and informal caregivers) and the older person themselves, where appropriate and necessary. For example, an intervention may be triggered when a condition, for example increased loneliness, is likely to occur. As a result, care providers may be brought through a decision making process of asking the individual whether they would like to engage with local service providers (such as being enrolled in a local choir). Additionally, the system may be part of an educational and interventional system which informs the person of their recent activities and encourages positive behaviours. For example after identifying periods of increased loneliness, the system may present the person with supportive and encouraging options such as "You haven't met your friends at choir over the last two weeks, would you like to attend choir practice tonight?"

\section{REFERENCES}

[1] K. McMorrow and R. Werner, The economic consequences of ageing populations, Economic Papers, 138, 1999.

[2] B.R. Greene, A. ODonovan, R. Romero-Ortuno, L. Cogan, C. Ni Scanaill and R.A. Kenny, Quantitative falls risk assessment using the timed up and go test, IEEE Trans. Biomed. Eng. Vol. 57(12), pp. 2918-26, 2010.

[3] M. Mulvenna, W. Carswell, P.J. McCullagh, J.C. Augusto, H. Zheng, P. Jeffers, H.Y. Wang and S. Martin, Visualization of Data for Ambient Assisted Living Services, IEEE Communications Magazine vol. 49 (1), pp. 110-117, 2011.

[4] M. Skubic, R. Guevara and M. Rantz, M, Testing classifiers for embedded health assessment, Impact Analysis of Solutions for Chronic Disease Prevention and Management, Vol. 7251, pp. 198-205, 2012.

[5] S. Helal, W. Mann, H. El-Zabadani, J. King, Y. Kaddoura, and E. Jansen, The gator tech smart house: A programmable pervasive space, Computer, Vol. 38(3), pp. 50-60, 2005.

[6] D. Cook, M. Schmitter-Edgecombe, A. Crandall, C. Sanders, and B. Thomas. Collecting and disseminating smart home sensor data in the CASAS project, Proceedings of the CHI Workshop on Developing Shared Home Behavior Datasets to Advance HCI and Ubiquitous Computing Research, 2009.

[7] C. Chen, B. Das, and D. Cook. A data mining framework for activity recognition in smart environments, Proceedings of the International Conference on Intelligent Environments, 2010.

[8] P. Rashidi, D.J. Cook, L.B. Holder and M. Schmitter-Edgecombe, Discovering Activities to Recognize and Track in a Smart Environment IEEE Trans. Knowledge and Data Engineering, vol.23, no.4, pp.527-539, 2011.

[9] H. Jimison and R. Bajcsy, Integrated Communications and Inference Systems for Continuous Coordinated Care of Older Adults in the Home, NSF Collaborative Research Grant. Accessed on: 12th Dec. 2012 http://www.orcatech.org/research/studies/integrated-communicationsand-inference-systems-for-continuous-coordinated-care-of-older-adultsin-the-home

[10] J.A. Kaye, S.A. Maxwell, N. Mattek, T. Hayes, Dodge H, Pavel M, Jimison H, Wild K, Boise L, and Zitzelberger T, Intelligent Systems for Assessing Aging Changes: Home-Based, Unobtrusive and Continuous Assessment of Aging, Journal of Gerontology: Psychological Sciences, Vol. 66B(suppl 1): ppi180-i190, 2011.

[11] S. Robben and B. Krose, Longitudinal Residential Ambient Monitoring Correlating Sensor Data to Functional Health Status, 7th International Conference on Pervasive Computing Technologies for Healthcare (PervasiveHealth), Italy, 2013

[12] A. Kealy, K. McDaid, J. Loane, L. Walsh, and J. Doyle, J, Derivation of night time behaviour metrics using ambient sensors, 7 th International Conference on Pervasive Computing Technologies for Healthcare (PervasiveHealth), Italy, 2013.

[13] T. Hastie, R. Tibshirani, and J.H. Friedman. The Elements of Statistical Learning. Springer-Verlag, 2001.
[14] J. Doyle, L. Walsh, A. Sassu and T. McDonagh, Designing a Wellness Self-Management Tool for Older Adults Results from a Field Trial of YourWellness, 8th International Conference on Pervasive Computing Technologies for Healthcare (PervasiveHealth), Germany, 2014. 